\title{
Event-Triggered Control Using a Positive Systems Approach
}

\author{
Frédéric Mazenc, Michael Malisoff, Corina Barbalata, and Zhong-Ping Jiang
}

\begin{abstract}
We provide a new event-triggered control strategy that relies on the notion of positive systems. Our results cover output feedback, and robustness with respect to uncertain dynamics. Our proofs are based on interval observers. Our example shows potential advantages of our approach over earlier event-triggered methods.
\end{abstract}

\section{INTRODUCTION}

Event-triggered control has played an increasingly important role in control theoretic research; see, for instance, the works [3], [11], [14], and [15]. One advantage of using event-triggered control is that it can reduce the computational burden associated with implementing controls, by only changing the value of the control when there is a significant event. These events can be characterized as times when the state enters some prescribed region of the state space. This contrasts with standard zero-order hold strategies, where the times at which the control values are recomputed are usually independent of the state. Much event-triggered control literature is based on small gain methods; see, e.g., [6]. Small gain methods have the desirable advantage of ensuring robustness to uncertainty, but can sometimes be conservative, insofar that they can lead to unnecessarily frequent recomputation times for the controls.

Although emerging computing methods can facilitate recomputing control values, the increasing use of shared wireless (or shared wired) networked control systems calls for designing controls that take computation, communication, and energy constraints into account [3]. This led to systematic designs for event-triggered controls, e.g., in [4], [5], [14], and [16]. During the same time, much research has been done on positive systems, i.e.,

Key Words: stabilization, event-triggered, interval observer.

F. Mazenc is with Inria Saclay, L2S-CNRS-CentraleSupélec, 3 rue Joliot Curie, 91192, Gif-sur-Yvette, France (e-mail: frederic.mazenc@12s.centralesupelec.fr).

M. Malisoff is with Department of Mathematics, Louisiana State University, Baton Rouge, LA 70803-4918, USA (e-mail: malisoff@1su.edu)

C. Barbalata is with Department of Mechanical and Industrial Engineering, Louisiana State University, Baton Rouge, LA 70803, USA (email: cbarbalata@1su.edu)

Z.-P. Jiang is with Department of Electrical and Computer Engineering, Tandon School of Engineering, New York University, Brooklyn, NY 11201, USA (email: zjiang@nyu.edu) systems for which the nonnegative orthant is positively invariant, leading to new control analysis and designs that overcome some of the challenges of using traditional Lyapunov function methods. Much work on positive systems uses interval observers (as defined, e.g., in [1], [9], and [12]), which provide intervals containing the values of unknown states when the inequalities involving vector valued solutions are viewed componentwise; see [9] and [10]. Positive systems and interval observers led to advances in aerospace engineering, mathematical biology, and other areas. However, we believe that these approaches had not been used for the systematic design and analysis of event-triggered controls.

This motivates this work, where we use a new positivity based event-triggered control technique to design control strategies that yield less conservative triggering conditions than those in the literature. We establish an output and a robustness result, covering cases where there may be time-varying uncertainties in the dynamics. Our stability proofs use interval observers as comparison systems, and are reminiscent of the results of [10] insofar that they are based on linear Lyapunov functions for positive systems. However, [10] did not cover eventtriggering, and to the best of our knowledge, the present work is the first systematic use of interval observers and positive systems to design event-triggered controls.

We provide the necessary notation and preliminaries and introduce our main class of systems in Section II Then, in Section III] we prove our theorems on stability, extensions to systems with outputs where the eventtriggered controller uses an observer, and robustness to uncertain models. In Section IV, we illustrate potential advantages of our method using an example where our method ensures less frequent control recomputations than the main small gain event-triggered result in [6]. We close in Section $V$ by summarizing our findings and suggesting future research directions.

\section{Preliminaries}

We use the following notation, where the dimensions of our Euclidean spaces are arbitrary unless otherwise noted. The arguments of functions will be omitted whenever no confusion can arise from the context. Set 
$\mathbb{Z}_{0}=\{0,1,2, \ldots\}$ and $\mathbb{N}=\mathbb{Z}_{0} \backslash\{0\}$. For a matrix $G=\left[g_{i j}\right] \in \mathbb{R}^{r \times s}$, we set $|G|=\left[\left|g_{i j}\right|\right]$, i.e., the entries of $|G|$ are the absolute values of the corresponding entries of $G$. Similarly, we set $G^{+}=\left[\max \left\{g_{i j}, 0\right\}\right]$ and $G^{-}=G^{+}-G$. A square matrix is called Metzler provided all of its off diagonal entries are nonnegative. For two matrices $D=\left[d_{i j}\right]$ an $E=\left[e_{i j}\right]$ of the same size, we write $D<E$ (resp., $D \leq E$ ) provided $d_{i j}<e_{i j}$ (resp., $d_{i j} \leq e_{i j}$ ) for all $i$ and $j$. We also write $D \nless E$ provided there is a pair $(i, j)$ such that $d_{i j}>e_{i j}$. We adopt similar notation for vectors. A matrix $S$ is called positive provided $0<S$. Let $\|\cdot\|$ denote the standard Euclidean norm of vectors and matrices, and $\|\cdot\|_{\infty}$ (resp., $\|\cdot\|_{J}$ ) denote the sup norm of matrix valued functions in this norm over their entire domain (resp., over an interval $J$ in their domain). We let $I$ denote the identity matrix of any dimension, and we use the standard definition and properties of state transition matrices (i.e., fundamental solutions), e.g., from [13, Appendix C].

We consider the system

$$
\dot{x}(t)=A x(t)+B u(t)
$$

where $x$ is valued in $\mathbb{R}^{n}, u$ is valued in $\mathbb{R}^{p}$, and the matrices $A$ and $B$ are constant. We assume:

Assumption 1: There is a matrix $K \in \mathbb{R}^{p \times n}$ such that the matrix $H=A+B K$ is Hurwitz and Metzler.

Remark 1: Assumption 11 is not restrictive because many systems satisfy it after a preliminary change of coordinates. When the pair $(A, B)$ is controllable, there is a change of coordinates that provides new matrices $A$ and $B$ that satisfy this assumption. In fact, under Assumption 11, there is a matrix $K \in \mathbb{R}^{p \times n}$ such that $A+B K$ is Hurwitz with negative real eigenvalues. Then there exists an invertible matrix $P \in \mathbb{R}^{n \times n}$ such that $P(A+B K) P^{-1}$ is Metzler because the Jordan canonical form of the matrix $A+B K$ is Metzler. Thus Assumption 1 is satisfied by the pair $\left(P A P^{-1}, P B\right)$.

Recall that the Zeno phenomenon is that a system with sampling has infinitely many sample times on some interval of finite length. A key ingredient in our analysis in later sections will be finding a lower bound $\nu>0$ on the intersample times $t_{i+1}-t_{i}$ between the event triggering times, which will imply that the Zeno phenomenon does not occur. This constant $\nu$ will be provided by the following lemma:

Lemma 1: Let $(A, B)$ satisfy Assumption 11. Let $\Gamma \in$ $\mathbb{R}^{n \times n}$ be a positive matrix such that the matrix

$$
M=H+|B K| \Gamma
$$

is Metzler and Hurwitz. Let $\Omega:[0,+\infty) \rightarrow \mathbb{R}^{n \times n}$ be the function

$$
\Omega(s)=e^{s A}+\int_{0}^{s} e^{\ell A} \mathrm{~d} \ell B K .
$$

Then there exists a constant $\nu>0$ such that for all $s \in[0, \nu]$, the matrix $\Omega(s)$ is invertible and the inequality

$$
\left|I-\Omega(s)^{-1}\right| \leq \Gamma
$$

is satisfied.

Proof: First observe that Assumption 1 implies that there exists a positive matrix $\Gamma \in \mathbb{R}^{n \times n}$ such that $M$ as defined in (2) is Metzler and Hurwitz. In fact, since $0 \leq|B K|$ and $0 \leq \Gamma$, it follows that $0 \leq|B K| \Gamma$, which implies that $M$ is Metzler. Moreover it is Hurwitz when the entries of $|B K| \Gamma$ are sufficiently small. Next, we conclude the proof by observing that $\Omega$ is continuous, $\Omega(0)=I$, and $0<\Gamma$.

Remark 2: Since $e^{s A}$ is invertible, the matrix $\Omega(s)$ in (3) with $s \geq 0$ is invertible if and only if $I+$ $\int_{0}^{s} e^{(\ell-s) A} \mathrm{~d} \ell B K \bar{K}$ is invertible. Thus, it is invertible if

$$
\left\|\int_{0}^{s} e^{(\ell-s) A} \mathrm{~d} \ell B K\right\|<1
$$

which follows by checking that the nullspace of $I+$ $\int_{0}^{s} e^{(\ell-s) A} \mathrm{~d} \ell B K$ is trivial. The inequality $\sqrt{5}$ ) is satisfied if $B K=0$. If $B K \neq 0$, then for any $\epsilon_{0} \in(0,1)$, we obtain the bound $s<s_{*}$, where $s_{*}$ is the sup of all $r$ values such that the integral in (5) is bounded above by $\epsilon_{0}$ for all $s \in[0, r]$. A more explicit bound on the allowable $s$ values in the $B K \neq 0$ case can be obtained as follows. If $B K \neq 0$, then [5] is satisfied if

$$
\int_{0}^{s} e^{(s-\ell)\|A\|} \mathrm{d} \ell<\frac{1}{\|B K\|} .
$$

If $A=0$, then this gives the condition $s<1 /\|B K\|$. On the other hand, if $A \neq 0$, then we instead have the condition $\left(e^{s\|A\|}-1\right) /\|A\|<1 /\|B K\|$ which is equivalent to $s<(1 /\|A\|) \ln (1+\|A\| /\|B K\|)$.

Remark 3: Notice for later use that since $M$ defined in (2) is Metzler and Hurwitz, there exist a vector $V \in$ $\mathbb{R}^{n}$ such that $0<V$, and a constant $p \in(0,+\infty)$, such that the inequality

$$
V^{\top} M \leq-p V^{\top}
$$

holds. This result is a consequence of [2, Lemma 2.3, p. 41], for instance.

\section{MAIN RESUlts}

\section{A. State Feedback Event-Triggered Control}

We next prove the following theorem, whose condition (c) means that for each $\epsilon>0$, there exist $t_{\ddagger} \in$ $\left(t_{i+1}, t_{i+1}+\epsilon\right)$ and $j \in\{1, \ldots, n\}$ such that

$$
\left|x_{j}\left(t_{\ddagger}\right)-x_{j}\left(t_{i}\right)\right|>\sum_{g=1}^{n} \Gamma_{j g}\left|x_{g}\left(t_{\ddagger}\right)\right|
$$

is satisfied, and where we continue our notation $|S|=$ $\left[\left|s_{i j}\right|\right]$ for matrices $S=\left[s_{i j}\right]$ from Section II] 
Theorem 1: Consider the system (1) under Assumption 1 and let $\Gamma=\left[\Gamma_{j, k}\right]>0$ satisfy the requirements of Lemma 11 and $K$ satisfy the requirements from Assumption 11. Consider the sequence of nonnegative numbers $t_{i}$ defined by $t_{0}=0$,

(a) $\dot{x}(t)=A x(t)+B K x\left(t_{i}\right)$ if $t \in\left[t_{i}, t_{i+1}\right)$,

(b) $\left|x(t)-x\left(t_{i}\right)\right| \leq \Gamma|x(t)|$ if $t \in\left[t_{i}, t_{i+1}\right)$, and

(c) For each $\epsilon>0$ and each $i \in \mathbb{Z}_{0}$ such that $t_{i+1}<$ $+\infty$, there is a $t_{\ddagger} \in\left(t_{i+1}, t_{i+1}+\epsilon\right)$ such that $\left|x\left(t_{\ddagger}\right)-x\left(t_{i}\right)\right| \nless \Gamma\left|x\left(t_{\ddagger}\right)\right|$

for all $i \in \mathbb{Z}_{0}$. Then the closed loop system given by (a)(c) admits the origin as a globally exponentially stable equilibrium point on $\mathbb{R}^{n}$.

Proof: First Part. Letting $\nu$ be the constant from Lemma 1], we prove that the inter-event times $t_{i+1}-t_{i}$ are bounded below by $\nu$. More precisely, we prove that either there are only a finite number of instants $t_{0}, \ldots, t_{j}$ and $\inf _{l \in\{0, \ldots, j-1\}}\left(t_{l+1}-t_{l}\right) \geq \nu$; or else there are infinitely many instants $t_{i}$ and $\inf _{l \in \mathbb{Z}_{0}}\left(t_{l+1}-t_{l}\right) \geq \nu$.

Consider any $i \in \mathbb{Z}_{\geq 0}$ such that the Zeno phenomenon does not occur between $\left[0, t_{i}\right]$. If $x\left(t_{i}\right)=0$, then the theorem of existence and uniqueness of the solutions implies that $x(t)=0$ for all $t \geq t_{i}$. Next, consider the case where $x\left(t_{i}\right) \neq 0$. Continuity of $x(t)$ implies that $t_{i+1}$ is well-defined and $t_{i+1}=+\infty$ or $t_{i+1}$ is finite and $t_{i+1}>t_{i}$. Consider the case where $t_{i+1}$ is finite. By integrating the equation in (a) between $\left[t_{i}, t\right)$ with $t \in\left[t_{i}, t_{i+1}\right)$, we obtain

$$
x(t)=\Omega\left(t-t_{i}\right) x\left(t_{i}\right)
$$

where $\Omega$ is defined in (3). From (4), it follows that, for any $t \in\left[t_{i}, t_{i}+\nu\right]$, the vector inequality $\left|I-\Omega\left(t-t_{i}\right)^{-1}\right||x(t)| \leq \Gamma|x(t)|$ is satisfied. It follows that

$$
\left|x(t)-\Omega\left(t-t_{i}\right)^{-1} x(t)\right| \leq \Gamma|x(t)| .
$$

This inequality in combination with 9 gives

$$
\left|x(t)-x\left(t_{i}\right)\right| \leq \Gamma|x(t)|
$$

for all $t \in\left[t_{i}, t_{i}+\nu\right]$. We conclude that $t_{i+1} \geq t_{i}+\nu$. Thus, the Zeno phenomenon does not occur and that the inter-sample times are bounded below by $\nu$

Second Part. We study the stability of the closed loop system from (a)-(c). The case where there is $j \in \mathbb{Z}_{\geq 0}$ such that $x\left(t_{j}\right)=0$ is trivial. Thus, we consider the case where $x\left(t_{j}\right) \neq 0$ for all $j \in \mathbb{Z}_{\geq 0}$. For notational convenience, we introduce the function $\mu$ defined by $\mu(t)=x\left(t_{i}\right)-x(t)$ for all $t \in\left[t_{i}, t_{i+1}\right)$ and all $i \in \mathbb{Z}_{0}$. Then (a)-(c) give

$$
\begin{aligned}
\dot{x}(t) & =H x(t)+B K \mu(t) \\
|\mu(t)| & \leq \Gamma|x(t)|
\end{aligned}
$$

for all $t \geq 0$, where $H=A+B K$ as before.
To study (12), we exploit the fact that $H$ is Metzler, to adopt an analysis approach based on interval observers. We introduce the dynamic extension

$$
\left\{\begin{array}{l}
\dot{\bar{x}}(t)=H \bar{x}(t)+(B K \mu(t))^{+} \\
\dot{\dot{x}}(t)=H \underline{x}(t)-(B K \mu(t))^{-}
\end{array} .\right.
$$

Consider a solution of $(12)$ with $x(0) \in \mathbb{R}^{n}$ as its initial condition and any initial conditions $\bar{x}(0) \in \mathbb{R}^{n}$ and $\underline{x}(0) \in \mathbb{R}^{n}$ for $[13$ such that

$$
\begin{gathered}
\underline{x}(0)<x(0)<\bar{x}(0) \\
\underline{x}(0)<0<\bar{x}(0)
\end{gathered}
$$

Observe that $\bar{e}(t)=\bar{x}(t)-x(t)$ and $\underline{e}(t)=x(t)-\underline{x}(t)$ satisfy

$$
\left\{\begin{array}{l}
\dot{\bar{e}}(t)=H \bar{e}(t)+(B K \mu(t))^{-} \\
\dot{e}(t)=H \underline{e}(t)+(B K \mu(t))^{+}
\end{array} .\right.
$$

Since the matrix $H$ is Metzler, it follows (e.g., from [8, Lemma 1]) that $\bar{e}(t) \geq 0$ and $\underline{e}(t) \geq 0$ for all $t \geq 0$. We deduce that

$$
\underline{x}(t) \leq x(t) \leq \bar{x}(t)
$$

and also

$$
\underline{x}(t) \leq 0 \leq \bar{x}(t)
$$

for all $t \geq 0$, where (17) follows because $\bar{x}$ and $-\underline{x}$ are solutions of $\dot{z}=H z+(B K \mu)^{ \pm}$and from again applying [8, Lemma 1] using the Metzler matrix $H$. We deduce from 16-17) that $\underline{x}(t)-\bar{x}(t) \leq x(t) \leq \bar{x}(t)-\underline{x}(t)$, i.e.,

$$
|x(t)| \leq s(t)
$$

for all $t \geq 0$, where $s(t)=\bar{x}(t)-\underline{x}(t)$.

We next analyze the behavior of $s$. We have

$$
\begin{aligned}
\dot{s}(t) & =H s(t)+(B K \mu(t))^{+}+(B K \mu(t))^{-} \\
& =H s(t)+\mid B K \mu(t)) \mid .
\end{aligned}
$$

Consider the linear function $W(s)=V^{\top} s$, where $V$ is the vector in Remark 3 The time derivative of $W$ along (19) satisfies

$$
\begin{aligned}
\dot{W}(t) & \left.=V^{\top} H s(t)+V^{\top} \mid B K \mu(t)\right) \mid \\
& \leq V^{\top} H s(t)+V^{\top}|B K| \Gamma|x(t)|,
\end{aligned}
$$

where the last inequality is a consequence of the inequality in (12). Substituting (18) in 20, we obtain

$$
\begin{aligned}
\dot{W}(t) & \leq V^{\top} H s(t)+V^{\top}|B K| \Gamma s(t) \\
& =V^{\top} M s(t),
\end{aligned}
$$

where $M$ is the matrix defined in (2). From (7), it follows that

$$
\dot{W}(t)=-p V^{\top} s(t)=-p W(s(t)) .
$$

Since $V>0$ and $s(t) \geq 0$ for all $t \geq 0$, we deduce that $s(t)$ exponentially converges to zero as $t \rightarrow+\infty$. We can assume that $s(0) \leq 4|x(0)|$, e.g., by requiring that $\bar{x}(0) \leq 2|x(0)|$ and $\underline{x}(0) \geq-2|x(0)|$. Hence, from the inequality 18 , we deduce that $x(t)$ goes exponentially to the origin as $t \rightarrow+\infty$. 


\section{B. Output Feedback Control}

The event-triggered control in Theorem 1 makes essential use of measurements of the state $x(t)$, and it is nontrivial to generalize it to cases where the state is not available for measurement. This motivates this section, where we consider the system (1) with an output, namely,

$$
\left\{\begin{array}{l}
\dot{x}(t)=A x(t)+B u(t) \\
y(t)=C x(t)
\end{array}\right.
$$

with $y$ valued in $\mathbb{R}^{q}$ and $C$ being a constant matrix. We use this classical assumption:

Assumption 2: The pair $(A, C)$ is observable.

Assumption 2 ensures that there is a matrix $L \in \mathbb{R}^{n \times q}$ such that the matrix

$$
G=A+L C
$$

is Hurwitz. This allows us to prove:

Theorem 2: Consider the system (23) under Assumptions 1,2 Let $\Gamma>0$ satisfy the requirements of Lemma 1 such that (7) holds, and the constant $\nu>0$ satisfy the requirements of Lemma 1 . Consider the sequence of nonnegative numbers $t_{i}$ and the feedback $K \hat{x}\left(t_{i}\right)$ defined by $t_{0}=0$,

(a') $\dot{x}(t)=A x(t)+B K \hat{x}\left(t_{i}\right)$ if $t \in\left(t_{i}, t_{i+1}\right)$,

(b') $\dot{\hat{x}}(t)=A \hat{x}(t)+B K \hat{x}\left(t_{i}\right)+L[C \hat{x}(t)-y(t)]$ if $t \in\left(t_{i}, t_{i+1}\right)$,

(c') $\left|\hat{x}(t)-\hat{x}\left(t_{i}\right)\right| \leq \Gamma|\hat{x}(t)|+\mathcal{B} \int_{\max \{0, t-\nu\}}^{t} \mid C \hat{x}(\ell)-$ $y(\ell) \mid \mathrm{d} \ell$ for all $t \in\left[t_{i}, t_{i+1}\right)$, and

(d') For each $\epsilon>0$ and each $i$ such that $t_{i+1}<+\infty$, there is a $t_{\ddagger} \in\left(t_{i+1}, t_{i+1}+\epsilon\right)$ such that $\mid \hat{x}\left(t_{\ddagger}\right)-$ $\hat{x}\left(t_{i}\right)|\nless \Gamma| \hat{x}\left(t_{\ddagger}\right)\left|+\mathcal{B} \int_{\max \left\{0, t_{\ddagger}-\nu\right\}}^{t_{\ddagger}}\right| C \hat{x}(\ell)-$ $y(\ell) \mid \mathrm{d} \ell$

with $i \in \mathbb{Z}_{0}$, where $\hat{x}(0) \neq 0$ and

$$
\mathcal{B}=\sup _{\ell \in[0, \nu]}\left|\Omega(\ell)^{-1}\right| e^{\nu|A|}|L| .
$$

Then the system given by (a')-(d') admits the origin as a globally exponentially stable equilibrium point on $\mathbb{R}^{n}$.

Proof: First part. We prove that the inter-event times $t_{i+1}-t_{i}$ are bounded below by $\nu$. More precisely, we prove that either there are only a finite number of instants $t_{0}, \ldots, t_{j}$ and

$$
\inf _{l \in\{0, \ldots, j-1\}}\left(t_{l+1}-t_{l}\right) \geq \nu
$$

or there are an infinite number of instants $t_{i}$ and $\inf _{l \in \mathbb{Z}_{0}}\left(t_{l+1}-t_{l}\right) \geq \nu$.

Let us consider any $i \in \mathbb{N}$ such that the Zeno phenomenon does not occur between $\left[0, t_{i}\right]$. If

$$
\Gamma\left|\hat{x}\left(t_{i}\right)\right|+\mathcal{B} \int_{\max \left\{0, t_{i}-\nu\right\}}^{t_{i}}|C \hat{x}(\ell)-y(\ell)| \mathrm{d} \ell=0,
$$

then $\hat{x}\left(t_{i}\right)=0$ and $C \hat{x}(\ell)=C x(\ell)$ for all $\ell \in\left[t_{i}-\delta_{0}, t_{i}\right]$ for some $\delta_{0}>0$ because $t_{i} \geq t_{1}>0$ (which follows because $\hat{x}(0) \neq 0)$. Assumption 2 implies that $x\left(t_{i}\right)=$ $\hat{x}\left(t_{i}\right)$. We deduce that $\left(x\left(t_{i}\right), \hat{x}\left(t_{i}\right)\right)=(0,0)$. Then the existence and uniqueness property of the solutions imply that $(x(t), \hat{x}(t))=(0,0)$ for all $t \geq t_{i}$.

Next, consider the case where

$$
\Gamma\left|\hat{x}\left(t_{i}\right)\right|+\mathcal{B} \int_{\max \left\{0, t_{i}-\nu\right\}}^{t_{i}}|C \hat{x}(\ell)-y(\ell)| \mathrm{d} \ell>0 .
$$

Continuity of $x$ implies that $t_{i+1}$ is well-defined, and that either $t_{i+1}=+\infty$, or else $t_{i+1}$ is finite and $t_{i+1}>t_{i}$. Consider the case where $t_{i+1}$ is finite. By integrating the $\hat{x}$-subsystem in (b') between $\left[t_{i}, t\right)$ with $t \in\left[t_{i}, t_{i+1}\right)$, we obtain

$$
\begin{aligned}
& \hat{x}\left(t_{i}\right)=\Omega\left(t-t_{i}\right)^{-1} \hat{x}(t) \\
& -\Omega\left(t-t_{i}\right)^{-1} \int_{t_{i}}^{t} e^{(t-\ell) A} L[C \hat{x}(\ell)-y(\ell)] \mathrm{d} \ell .
\end{aligned}
$$

As an immediate consequence,

$$
\begin{aligned}
\left|\hat{x}(t)-\hat{x}\left(t_{i}\right)\right|= & \mid \hat{x}(t)-\Omega\left(t-t_{i}\right)^{-1} \hat{x}(t) \\
& +\Omega\left(t-t_{i}\right)^{-1} \int_{t_{i}}^{t} \Delta(t, \ell) \mathrm{d} \ell \mid \\
\leq & \left|I-\Omega\left(t-t_{i}\right)^{-1}\right||\hat{x}(t)| \\
& +\left|\Omega\left(t-t_{i}\right)^{-1} \int_{t_{i}}^{t} \Delta(t, \ell) \mathrm{d} \ell\right| \\
\leq & \Gamma|\hat{x}(t)| \\
& +\left|\Omega\left(t-t_{i}\right)^{-1} \int_{t_{i}}^{t} \Delta(t, \ell) \mathrm{d} \ell\right|
\end{aligned}
$$

when $t \in\left[t_{i}, t_{i}+\nu\right]$, where $\Delta(t, \ell)=e^{(t-\ell) A} L[C \hat{x}(\ell)-$ $y(\ell)]$. Consequently,

$$
\begin{aligned}
\left|\hat{x}(t)-\hat{x}\left(t_{i}\right)\right| \leq & \Gamma|\hat{x}(t)|+\mathcal{B} \int_{t_{i}}^{t} \hat{C}(\ell) \mathrm{d} \ell \\
\leq & \Gamma|\hat{x}(t)| \\
& +\mathcal{B} \int_{\max \{0, t-\nu\}}^{t} \hat{C}(\ell) \mathrm{d} \ell
\end{aligned}
$$

for all $t \in\left[t_{i}, t_{i}+\nu\right]$, where $\hat{C}(\ell)=|C \hat{x}(\ell)-y(\ell)|$. It follows that $t_{i+1}-t_{i} \geq \nu$ for all $i \in \mathbb{Z}_{0}$.

Second part. In this part, we prove the exponential stability of the equilibrium point of the closed loop system. In what follows, all equalities and inequalities should be understood to hold for all $t \geq 0$, unless otherwise indicated.

$$
\text { Let } \tilde{x}(t)=\hat{x}(t)-x(t) \text {. Then }
$$$$
\dot{\tilde{x}}(t)=G \tilde{x}(t)
$$

where $G$ is the matrix defined in (24). Notice for later use that $\tilde{x}(t)$ exponentially converges to the origin as $t \rightarrow+\infty$. Also, with the simplifying notation $\hat{\mu}(t)=$ $\hat{x}\left(t_{i}\right)-\hat{x}(t)$, we have

$$
\dot{\hat{x}}(t)=H \hat{x}(t)+B K \hat{\mu}(t)+L C \tilde{x}(t)
$$

We next introduce this comparison system:

$$
\left\{\begin{array}{l}
\dot{\bar{x}}(t)=H \bar{x}(t)+(B K \hat{\mu}(t))^{+}+(L C \tilde{x}(t))^{+} \\
\dot{\dot{x}}(t)=H \underline{x}(t)-(B K \hat{\mu}(t))^{-}-(L C \tilde{x}(t))^{-}
\end{array}\right.
$$


As in the previous section, one can prove that if $\underline{x}(0)<$ $0<\bar{x}(0)$ and $\underline{x}(0)<\hat{x}(0)<\bar{x}(0)$, then for all $t \geq 0$, the inequalities

$$
\underline{x}(t) \leq 0 \leq \bar{x}(t) \text { and } \underline{x}(t) \leq \hat{x}(t) \leq \bar{x}(t)
$$

hold. For the preceding initial conditions, consider $s(t)=\bar{x}(t)-\underline{x}(t)$. Then the bounds 35 give

$$
|\hat{x}(t)| \leq s(t) \text {. }
$$

Simple calculations give

$$
\dot{s}(t)=H s(t)+|B K \hat{\mu}(t)|+|L C \tilde{x}(t)| .
$$

Consider the linear function $W(s)=V^{\top} s$. Then, for all $t \geq 0$, 31 and our choice (2) of $M$ give

$$
\begin{aligned}
\dot{W}(t)= & V^{\top} H s(t)+V^{\top}|B K \hat{\mu}(t)| \\
& +V^{\top}|L C \tilde{x}(t)| \\
\leq & V^{\top} H s(t)+V^{\top}|B K|(\Gamma|\hat{x}(t)| \\
& \left.+\mathcal{B} \int_{\max \{0, t-\nu\}}^{t} \hat{C}(\ell) \mathrm{d} \ell\right) \\
& +V^{\top}|L C \tilde{x}(t)| \\
= & V^{\top} M s(t)+V^{\top}|L C \tilde{x}(t)| \\
& +V^{\top}|B K| \mathcal{B} \int_{\max \{0, t-\nu\}}^{t} \hat{C}(\ell) \mathrm{d} \ell .
\end{aligned}
$$

Using (7), we obtain

$$
\begin{aligned}
\dot{W}(t) \leq & -p W(s(t))+V^{\top}|L C \tilde{x}(t)| \\
& +V^{\top}|B K| \mathcal{B} \int_{\max \{0, t-\nu\}}^{t} \hat{C}(\ell) \mathrm{d} \ell \\
= & -p W(s(t))+\Xi(t),
\end{aligned}
$$

where

$$
\begin{aligned}
\Xi(t)= & V^{\top}|B K| \mathcal{B}|C| \int_{\max \{0, t-\nu\}}^{t}|\tilde{x}(\ell)| \mathrm{d} \ell \\
& +V^{\top}|L C \tilde{x}(t)| .
\end{aligned}
$$

Since $s(t) \geq 0$ for all $t \geq 0$ and $\Xi$ is nonnegative valued and converges exponentially to the origin as $t \rightarrow+\infty$, it follows that $W(s(t))$ converges exponentially to zero as $t \rightarrow+\infty$. Hence, $s(t)$ converges exponentially to the origin as $t \rightarrow+\infty$. The theorem now follows from (36), the bound $|x(t)| \leq|\hat{x}(t)|+|\tilde{x}(t)|$, and the corresponding argument from the proof of Theorem 1 .

\section{Robustness}

We next generalize our result from the system (1) with known vector fields to cases with an additive disturbance of the form $A_{\delta}(t) x(t)$ of the form

$$
\dot{x}(t)=\left(A+A_{\delta}(t)\right) x(t)+B u
$$

where the function $A_{\delta}$ will satisfy our requirements below. We impose a small enough known bound $\left|A_{\delta}\right|_{\infty}$ on the unknown function, because our objective is to propose a stabilizing feedback that is robust with respect to the term $A_{\delta}(t) x(t)$. This motivates the following assumption, which will be satisfied under our Assumption 1 when the entries of $A_{\delta}$ and $\Gamma$ are small enough in absolute value and $A$ is Metzler with positive off diagonal elements:

Assumption 3: The bounded function $A_{\delta}$ : $[0,+\infty) \rightarrow \mathbb{R}^{n \times n}$ is piecewise continuous and such that $A+A_{\delta}(t)$ is Metzler for all $t \geq 0$. Also, for the Hurwitz matrix $H$ satisfying Assumption 1, and for a $\Gamma$ satisfying the requirements of Lemma 1 , there is a constant $a>0$ such that the system

$$
\dot{\xi}(t)=\left[H+\left|A_{\delta}(t)\right|+(1+a)|B K| \Gamma\right] \xi(t)
$$

admits the origin as a uniformly exponentially stable equilibrium point on $\mathbb{R}^{n}$.

We prove the following result:

Theorem 3: Let 41) satisfy Assumptions 1 and 3 and choose $\nu$ to satisfy Lemma 1 . Let $A_{\delta}$ be such that

$$
\begin{aligned}
& \left\|A_{\delta}\right\|_{\infty} \nu e^{\nu\left(\|A\|+\left\|A_{\delta}\right\|_{\infty}\right)}(1+\nu\|B K\|)\left\|\Omega^{-1}\right\|_{[0, \nu]} \\
& <c_{0}
\end{aligned}
$$

where $c_{0} \in(0,1)$ is any constant such that

$$
\begin{aligned}
& \frac{c_{0}}{1-c_{0}}\left\|\Omega^{-1}\right\|_{[0, \nu]} \leq \\
& a \min \left\{\Gamma_{i j}: 1 \leq i \leq n, 1 \leq j \leq n\right\} .
\end{aligned}
$$

Then the system with sampling times $t_{i}$ defined by $t_{0}=$ 0 ,

(a") $\dot{x}(t)=\left(A+A_{\delta}(t)\right) x(t)+B K x\left(t_{i}\right)$ if $t \in\left(t_{i}, t_{i+1}\right)$, (b") $\left|x(t)-x\left(t_{i}\right)\right| \leq(1+a) \Gamma|x(t)|$ if $t \in\left[t_{i}, t_{i+1}\right)$, and

(c") For each $\epsilon>0$ and each $i$ such that $t_{i+1}<+\infty$, there is a $t_{\ddagger} \in\left(t_{i+1}, t_{i+1}+\epsilon\right)$ such that $\mid x\left(t_{\ddagger}\right)-$ $x\left(t_{i}\right)|\nless(1+a) \Gamma| x\left(t_{\ddagger}\right) \mid$

for all $i \in \mathbb{Z}_{0}$ admits the origin as a globally exponentially stable equilibrium point on $\mathbb{R}^{n}$.

Proof: First part. We first prove that the interevent times $t_{i+1}-t_{i}$ are bounded below by $\nu$. More precisely, we prove that either there are only a finite number of instants $t_{0}, \ldots, t_{j}$ and $\inf _{l \in\{0, \ldots, j-1\}}\left(t_{l+1}-\right.$ $\left.t_{l}\right) \geq \nu$; or else there are an infinite number of instants $t_{i}$ and $\inf _{l \in \mathbb{Z}_{0}}\left(t_{l+1}-t_{l}\right) \geq \nu$. Our proof is based on [7, Lemmas 1-2], which we also include for reference in our appendix below.

Let $\Phi_{A+A_{\delta}}$ be the state transition matrix of $A+A_{\delta}(t)$. Then, for any $i \in \mathbb{Z}_{0}$, we can apply variation of parameters to (a") over $\left[t_{i}, t\right)$ with $t \in\left[t_{i}, t_{i+1}\right]$ to get

$$
\begin{aligned}
& x(t)= \\
& {\left[\Phi_{A+A_{\delta}}\left(t, t_{i}\right)+\int_{t_{i}}^{t} \Phi_{A+A_{\delta}}(t, \ell) \mathrm{d} \ell B K\right] x\left(t_{i}\right) .}
\end{aligned}
$$

For all $t \in\left[t_{i}, t_{i}+\nu\right]$, the quantity in squared brackets in 45 can be written as

$$
\begin{aligned}
& \Omega\left(t-t_{i}\right)+\Phi_{A+A_{\delta}}\left(t, t_{i}\right)-e^{A\left(t-t_{i}\right)} \\
& +\int_{t_{i}}^{t}\left(\Phi_{A+A_{\delta}}(t, \ell)-e^{(t-\ell) A}\right) \mathrm{d} \ell B K \\
& =\Omega\left(t-t_{i}\right)\left[I+\eta\left(t, t_{i}\right)\right],
\end{aligned}
$$


where

$$
\begin{aligned}
& \eta\left(t, t_{i}\right)=\Omega\left(t-t_{i}\right)^{-1}\left[\Phi_{A+A_{\delta}}\left(t, t_{i}\right)-e^{A\left(t-t_{i}\right)}\right. \\
& \left.+\int_{t_{i}}^{t}\left(\Phi_{A+A_{\delta}}(t, \ell)-e^{(t-\ell) A}\right) \mathrm{d} \ell B K\right]
\end{aligned}
$$

and $\Omega$ as defined in Lemma 1. Using the bound

$$
\begin{aligned}
& \left\|\Phi_{A+A_{\delta}}\left(t, t_{i}\right)-e^{A\left(t-t_{i}\right)}\right\| \leq \\
& \left\|A_{\delta}\right\| \nu e^{\nu\left(\|A\|+\left\|A_{\delta}\right\|_{\infty}\right)}
\end{aligned}
$$

which follows e.g., from [7, Lemma 2], and recalling [43, it follows that $\left\|\eta\left(t, t_{i}\right)\right\|<c_{0}<1$, and therefore that $I+\eta\left(t, t_{i}\right)$ is invertible, which implies that $x\left(t_{i}\right)=$ $\mathcal{R}\left(t, t_{i}\right) x(t)$ for all $t \in\left[t_{i}, t_{i+1}\right)$, where

$$
\begin{aligned}
& \mathcal{R}\left(t, t_{i}\right)= \\
& \left(\Phi_{A+A_{\delta}}\left(t, t_{i}\right)+\int_{t_{i}}^{t} \Phi_{A+A_{\delta}}(t, \ell) \mathrm{d} \ell B K\right)^{-1} .
\end{aligned}
$$

This is equivalent to $x(t)-x\left(t_{i}\right)=\left[I-\mathcal{R}\left(t, t_{i}\right)\right] x(t)$, which can be rewritten as

$$
\begin{aligned}
x(t)-x\left(t_{i}\right)= & {\left[I-\Omega\left(t-t_{i}\right)^{-1}+\Omega\left(t-t_{i}\right)^{-1}\right.} \\
& \left.-\mathcal{R}\left(t, t_{i}\right)\right] x(t)
\end{aligned}
$$

As an immediate consequence,

$$
\begin{aligned}
\left|x(t)-x\left(t_{i}\right)\right| \leq & {\left[\left|I-\Omega\left(t-t_{i}\right)^{-1}\right|\right.} \\
& \left.+\left|\Omega^{\sharp}\left(t, t_{i}\right)\right|\right]|x(t)| \\
\leq & {\left[\Gamma+\left|\Omega^{\sharp}\left(t, t_{i}\right)\right|\right]|x(t)| }
\end{aligned}
$$

where $\Omega^{\sharp}\left(t, t_{i}\right)=\Omega\left(t-t_{i}\right)^{-1}-\mathcal{R}\left(t, t_{i}\right)$ and the last inequality is a consequence of Lemma 1 . Also, with choice $\bar{c}=a \min _{i j} \Gamma_{i j}$, and for any matrix $S \in \mathbb{R}^{n \times n}$ that satisfies ||$S|| \leq \bar{c}$, we have $|S| \leq a \Gamma$. Specializing the preceding observation to the case where $S=\Omega(t-$ $\left.t_{i}\right)^{-1}-\mathcal{R}\left(t, t_{i}\right)$, it follows from Lemma A.1 in the appendix below (applied with $M_{0}=\Omega\left(t-t_{i}\right)$ and $N_{0}$ being the quantity in squared brackets in (47)) and (43)(44) give

$$
\begin{aligned}
& \left\|M_{0}^{-1}-\left(M_{0}+N_{0}\right)^{-1}\right\| \\
& =\left\|\Omega\left(t-t_{i}\right)^{-1}-\mathcal{R}\left(t, t_{i}\right)\right\| \leq \frac{\left\|\Omega^{-1}\right\|_{[0, \nu]} c_{0}}{1-c_{0}} \\
& \leq a \min \left\{\Gamma_{i j}: 1 \leq i \leq n, 1 \leq j \leq n\right\},
\end{aligned}
$$

and so also $\left|M_{0}^{-1}-\left(M_{0}+N_{0}\right)^{-1}\right| \leq a \Gamma$, by writing the quantity inside the inverse in (49) as $M_{0}+N_{0}$ and then noting that $\left\|M_{0}^{-1}|||| N_{0}\right\|<c_{0}$. Hence, by [51), the inequality $\left|x(t)-x\left(t_{i}\right)\right| \leq(1+a) \Gamma|x(t)|$ holds, which gives $t_{i+1} \geq t_{i}+\nu$.

Second part. We study the stability of (a")-(d"). We have

$$
\begin{aligned}
\dot{x}(t)= & \left(H+A_{\delta}(t)^{+}-A_{\delta}(t)^{-}\right) x(t) \\
& +B K \mu(t) \\
|\mu(t)| \leq & (1+a) \Gamma|x(t)|
\end{aligned}
$$

where $\mu(t)=x\left(t_{i}\right)-x(t)$. We introduce the interval observer

$$
\left\{\begin{aligned}
\dot{\bar{x}}(t)= & \left(H+A_{\delta}(t)^{+}\right) \bar{x}(t)-A_{\delta}(t)^{-} \underline{x}(t) \\
& +(B K \mu(t))^{+} \\
\dot{x}(t)= & \left(H+A_{\delta}(t)^{+}\right) \underline{x}(t)-A_{\delta}(t)^{-} \bar{x}(t) \\
& -(B K \mu(t))^{-} .
\end{aligned}\right.
$$

Then when the initial conditions are such that $\underline{x}(0)<$ $x(0)<\bar{x}(0), \underline{x}(0)<0<\bar{x}(0)$, the inequalities $\underline{x}(t)<$ $x(t)<\bar{x}(t), \underline{x}(t)<0<\bar{x}(t)$, and $|x(t)| \leq s(t)$ hold for all $t \geq 0$, where $s(t)=\bar{x}(t)-\underline{x}(t)$, by the corresponding argument from the second part of the proof of Theorem 2

Then 54) gives $\dot{s}(t)=\left(H+\left|A_{\delta}(t)\right|\right) s(t)+|B K \mu(t)|$. From the inequality in $(53)$, we deduce that there is a function $\mathcal{F}$ such that $0 \leq \mathcal{F}(t) \leq(1+a)|B K| \Gamma$ and $\dot{s}(t)=\left(H+\left|A_{\delta}(t)\right|+\mathcal{F}(t)\right) s(t)$ for all $t \geq 0$. Since $s(t)$ is nonnegative and $H+\left|A_{\delta}(t)\right|+\mathcal{F}(t)$ is Metzler for all $t \geq 0$, we deduce from Assumption 3 and the comparison principle that $s(t)$ converges exponentially to the origin as $t \rightarrow+\infty$, so since $|x(t)| \leq s(t)$ for all $t \geq 0$, and since we can assume as before that $s(0) \leq$ $4|x(0)|$, we conclude that $x(t)$ converges exponentially to the origin as $t \rightarrow+\infty$ as well.

\section{Illustration}

In this section, we illustrate Theorem 1. Consider the benchmark system

$$
\left\{\begin{array}{l}
\dot{x}_{1}(t)=x_{1}(t)+\frac{1}{2} x_{2}(t)+u \\
\dot{x}_{2}(t)=\frac{3}{2} x_{1}(t)+u
\end{array}\right.
$$

where $x_{1}$ and $x_{2}$ are valued in $\mathbb{R}$ and $u$ is the input. Then, using the notation $K=\left[\begin{array}{ll}K_{1} & K_{2}\end{array}\right]$, Assumption 1$]$ will be satisfied if

$$
H=\left[\begin{array}{cc}
1+K_{1} & \frac{1}{2}+K_{2} \\
\frac{3}{2}+K_{1} & K_{2}
\end{array}\right]
$$

is Hurwitz and Metzler. Since $H$ will be Hurwitz and Metzler if $K_{1}+K_{2}<-3 / 2, K_{1} \geq-3 / 2$, and $K_{2} \geq$ $-1 / 2$, it follows that Assumption 1 is satisfied using $K_{1}=-4 / 3$ and $K_{2}=-1 / 3$. Then, with the notation of Section III] we have

$$
\begin{aligned}
& H=\left[\begin{array}{rr}
-\frac{1}{3} & \frac{1}{6} \\
\frac{1}{6} & -\frac{1}{3}
\end{array}\right], B K=\left[\begin{array}{rr}
-\frac{4}{3} & -\frac{1}{3} \\
-\frac{4}{3} & -\frac{1}{3}
\end{array}\right], \\
& \text { and } M=H+|B K| \Gamma \\
& =\left[\begin{array}{rr}
-\frac{1}{3} & \frac{1}{6} \\
\frac{1}{6} & -\frac{1}{3}
\end{array}\right]+\left[\begin{array}{rr}
\frac{4}{3} & \frac{1}{3} \\
\frac{4}{3} & \frac{1}{3}
\end{array}\right] \Gamma .
\end{aligned}
$$

Then simple Mathematica calculations show that the assumptions of Lemma 1 and Theorem 1 are satisfied with all entries of $\Gamma=\left[\Gamma_{i j}\right]$ being $\Gamma_{i j}=0.045$ and the lower bound $\nu=0.122$ on the sampling intervals $t_{i+1}-t_{i}$. 
We next compare the preceding lower bound with one that can be obtained from [6, p.72, Theorem 5.2] using the small gain approach. Recall that in the linear time invariant case, the triggering times in [6, Theorem 5.2] are such that when $t_{i+1}$ is finite, it is the smallest time $t \geq t_{i}$ such that $\rho(\|x(t)\|)=\left\|x(t)-x\left(t_{i}\right)\right\|$, for any class $\mathcal{K}$ function $\rho$ such that $\rho(\gamma(s))<s$ for all $s>0$, where $\gamma \in \mathcal{K}_{\infty}$ is the overshoot function in a suitable input-to-state stability estimate for

$$
\dot{x}=(A+B K) x+B K w,
$$

i.e., there is a class $\mathcal{K} \mathcal{L}$ function $\beta$ such that along all solutions of (58) for all $t \geq 0$, we have $\|x(t)\| \leq$ $\max \left\{\beta(\|x(0)\|, t), \gamma\left(\|w\|_{\infty}\right)\right\}$; see [6] for the standard definitions of input-to-state stability and the classes $\mathcal{K}$, $\mathcal{K}_{\infty}$, and $\mathcal{K} \mathcal{L}$ of comparison functions. In the linear case, we can apply variation of parameters to 58 to show that the least conservative choice of $\gamma$ that satisfies the preceding requirements is

$$
\begin{aligned}
& \gamma(s)=2 \bar{M} s, \text { where } \\
& \bar{M}=\sup _{t \geq 0} \int_{0}^{t}\left|e^{\ell H} B K\right| \mathrm{d} \ell .
\end{aligned}
$$

Hence, the least conservative choice of $\rho$ is $\rho(s)=\frac{s}{2 M}$, which implies that when $t_{i+1}$ is finite, it is the supremum of the times $t>t_{i}$ such that

$$
\left\|I-\Omega^{-1}\left(t-t_{i}\right)\right\|<\frac{1}{2 \bar{M}},
$$

by 9 . Hence, the lower bound on the inter-sample times $t_{i+1}-t_{i}$ guaranteed in this case is the largest $q$ such that

$$
\left\|I-\Omega^{-1}(q)\right\| \leq \frac{1}{2 \bar{M}},
$$

which we computed to be $q=0.0838$, using Mathematica. Since this is significantly below the lower bound $\nu=0.122$ we obtained using our positive systems approach, this illustrates a potential advantage of our positive systems approach, namely, the ability of our method to ensure less frequent event-triggering times and therefore less frequent control recomputations.

Remark 4: Even in scalar cases, our approach from our Theorem 1 offers potential advantages over [6], in terms of ensuring a larger lower bound $\nu$ on the intersample times $\sup \left\{t_{i+1}-t_{i}\right\}$ as compared with [6]. For instance, consider the scalar case with $A=B=1$, $K=-2, H=-1$, and $\Gamma=\frac{1}{2} 0.9999$. Then, if we apply the same reasoning as we did in the preceding two-dimensional case, simple Mathematica calculations show that our requirements from Theorem 1 are satisfied using $\nu=0.2875$. On the other hand, the largest $\nu$ value for which the requirements from [6, p.72, Theorem 5.2] would be satisfied in this scalar case is 0.1823 . This again illustrates the considerable improvement that is made possible by our Theorem 1 .

\section{CONCLUSION}

We proposed a new triggered control design, where instead of small gain or other standard approaches, we used positive systems and interval observers. This allowed us to cover large classes of linear time invariant systems with outputs, and cases where there can be timevarying uncertainty in the coefficients of the systems. Our example illustrated how our method can lead to less conservative lower bounds on the inter-sampling times, compared with small gain approaches, and therefore ensure less frequent sampling. In our future work, We aim to extend our work to cover linear time-varying systems, whose coefficients can be expressed as sums of constant matrices and small enough time-varying parts. We aim to further extend our work to nonlinear systems.

\section{APPENDIX: LEMMAS FROM [7]}

We used the following lemmas (which are Lemmas 1-2 from [7], respectively) in our proof of Theorem 3 above:

Lemma A.1: Let $M_{0} \in \mathbb{R}^{n \times n}$ be an invertible matrix. Let $N_{0} \in \mathbb{R}^{n \times n}$ be a matrix. Let $\bar{n}$ and $\bar{m}$ be two constants such that $\left\|M_{0}^{-1}\right\| \leq \bar{m}$ and $\left\|N_{0}\right\| \leq \bar{n}$. Assume that

$$
\bar{m} \bar{n}<1 \text {. }
$$

Then the matrix $M_{0}+N_{0}$ is invertible and

$$
\left\|M_{0}^{-1}-\left(M_{0}+N_{0}\right)^{-1}\right\| \leq \frac{\bar{m}^{2} \bar{n}}{1-\bar{m} \bar{n}}
$$

is satisfied.

Lemma A.2: Let $\mathcal{A} \in \mathbb{R}^{n \times n}$ be a constant matrix. Consider the system

$$
\dot{\zeta}(t)=[\mathcal{A}+\mathcal{E}(t)] \zeta(t)
$$

where $\zeta$ is valued in $\mathbb{R}^{n}$ and $\mathcal{E}:[0,+\infty) \rightarrow \mathbb{R}^{n \times n}$ is a bounded locally Lipschitz function. Let $\phi$ denote the fundamental solution of the system A.3. Then for all $t_{1} \in \mathbb{R}$ and $t_{2} \in \mathbb{R}$ such that $t_{1} \geq t_{2}$, the inequality

$$
\begin{aligned}
& \left\|\phi\left(t_{1}, t_{2}\right)-e^{\mathcal{A}\left(t_{1}-t_{2}\right)}\right\| \\
& \leq\|\mathcal{E}\|_{\infty}\left(t_{1}-t_{2}\right) e^{\left(\|\mathcal{A}\|+\|\mathcal{E}\|_{\infty}\right)\left(t_{1}-t_{2}\right)}
\end{aligned}
$$

is satisfied.

\section{REFERENCES}

[1] Gouzé, J.-L., A. Rapaport, and Z. Hadj-Sadok. Interval observers for uncertain biological systems. Ecological Modelling, 133(12):45-56, 2000

[2] Haddad, W., V. Chellaboina, and Q. Hui. Nonnegative and Compartmental Dynamical Systems. Princeton University Press, Princeton, NJ, 2010.

[3] Heemels, W., K. Johansson, and P. Tabuada. An introduction to event-triggered and self-triggered control. In Proceedings of 51st IEEE Conference on Decision and Control (Maui, Hawaii, 10-13 December 2012), pp. 3270-3285. 
[4] Heemels, W., J. Sandee, and P. van den Bosch. Analysis of eventdriven controllers for linear systems. International Journal of Control, 81:571-590, 2008.

[5] Henningsson, T., E. Johannesson, and A. Cervin. Sporadic eventbased control of first-order linear stochastic systems. Automatica, 44(11):2890-2895, 2008.

[6] Jiang, Z.-P., and T. Liu. Small-gain theory for stability and control of dynamical networks: A survey. Annual Reviews in Control, 46:58-79, 2018.

[7] Mazenc, F., S. Ahmed, and M. Malisoff. Finite time estimation through a continuous-discrete observer. International Journal of Robust and Nonlinear Control, 28(16):4831-4849, 2018.

[8] Mazenc, F., V. Andrieu, and M. Malisoff. Design of continuousdiscrete observers for time-varying nonlinear systems. Automatica, 57(7):135-144, 2015.

[9] Mazenc, F., and O. Bernard. Interval observers for linear timeinvariant systems with disturbances. Automatica, 47(1):140-147, 2011.

[10] Mazenc, F., and M. Malisoff. Stability analysis for time-varying systems with delay using linear Lyapunov functionals and a positive systems approach. IEEE Transactions on Automatic Control, 61(3):771-776, 2016.

[11] Peralez, J., V. Andrieu, M. Nadri, and U. Serres. Event-triggered output feedback stabilization via dynamic high-gain scaling. IEEE Transactions on Automatic Control, 63(8):2537-2549, 2018.

[12] Raissi, T., D. Efimov, and A. Zolghadri. Interval state estimation for a class of nonlinear systems. IEEE Transactions on Automatic Control, 57(1):260-265, 2012.

[13] Sontag, E. Mathematical Control Theory, Second Edition. Springer, New York, NY, 1998.

[14] Tabuada, P. Event-triggered real-time scheduling of stabilizing control tasks. IEEE Transactions on Automatic Control, 52(9):1680-1685, 2007.

[15] Wang, X., and M. Lemmon. Self-triggered feedback control systems with finite-gain $L_{2}$ stability. IEEE Transactions on Automatic Control, 54(3):452-467, 2009.

[16] Yook, J., D. Tilbury, and N. Soparkar. Trading computation for bandwidth: Reducing communication in distributed control systems using state estimators. IEEE Transactions on Control Systems Technology, 10(4):503-518, 2002. 